\title{
XLV. A simple treatment of the secondary maxima of grating spectra
}

\section{R.W. Wood}

To cite this article: R.W. Wood (1907) XLV. A simple treatment of the secondary maxima of grating spectra, Philosophical Magazine Series 6, 14:82, 477-482, DOI: 10.1080/14786440709463706

To link to this article: http://dx.doi.org/10.1080/14786440709463706

曲 Published online: 16 Apr 2009.

Submit your article to this journal $₫$

Џll Article views: 2

Q View related articles $₫$ 
XLV. A Simple Treatment of the Secondary Masima of Grating Spectra. By R. W. Woon, Professor of Experimental Pliysics, Johns Hopkins University *.

THE usual method of treating the diffraction spectra produced by gratings is so involved, that the student is apt to lose all idea of the physical significance of the expressions. An elaborate formula, involving double integrals, the development of which requires several pages of pure mathematics, and is finally solved by graphical methods, shows that between the principal maxima produced by the grating, there are present $(n-2)$ secondary maxima, where $n$ is the number of lines of the grating. As an example we may take the case where the curves $x=n \tan z ; y=\tan n z$ are plotted, the secondary maxima being given by the points of intersection of the two curves. It has been my experience that students go through this treatment without having the faintest idea as to why secondary maxima are produced at all, though each step is fully understood from a purely mathematical standpoint.

A method occurred to me during a lecture last winter, by which the whole thing conld be discussed without any mathematics at all, the relative intensities of the principal and secondary maxima, their position and number being computed with the greatest ease, from most elementary principles. We shall make use of the well-known method of compounding vibrations, which is employed in the elementary development of Cornu's spiral, and shall show that we have minima equal to zero whenever the amplitude lines form a closed symmetrical figure, or mutually annul each other in pairs. The closed figures are either triangles, squares, regular polygons or star-shaped figures, which can be plotted in a very simple manner, described later on.

The method is somewhat similar to that employed by Kimbal (Phil. Mag. July 1903), whose discussion, however, is not always easily followed by students, and is not wholly devoid of mathematics.

It is so simple that I shall be surprised if others have not used it before, but I have found it so helpful myself, that I believe that every teacher of elementary or advanced optics ean employ it to advantage in class work, and that on this account it is worth putting on record.

Fraunhofer's treatment shows that a single slit produces maxima and minima, which recede from the centre and

* Communicated by the Author.

Phil. Mag. S.6. Vol. 14. No.82. Oct. 1907. $2 \mathrm{~K}$ 
broaden as the slit-width decreases. These he called spectra of the first class. In the case of the gratings used for optical purposes, the lines are so fine that the central maximum of the first-class spectra occupies the entire field; i. e. there are no minima, a single line scattering light of decreasing intensity throughout the entire range botween $0^{\circ}$ and $90^{\circ}$. In the present treatment we shall consider our lines of this degree of fineness. "Absent spectra," resulting from finite width of the line; and the consequent existence of first-class minima, can be separately dealt with.

We will consider parallel rays incident normally upon the grating, the parallel diffracted rays being brought to a focus by a lens.

Each line of the grating acting alone, we will suppose to produce unit amplitude at the focus.

We find the resultant amplitude produced by a number of lines operating together by the well-known device employed in the elementary treatment of Cornu's spiral, the resultant amplitude being the closing side of a polygon, the sides of which (vectors) represent the amplitudes and phases of the vibrations coming from the grating-lines. We can plot the intensity curve for a three-line grating, by considering phase differences (P.D.) which increase by $20^{\circ}$. In the normal direction (P.D. $0^{\circ}$ ) the intensity will be $3^{2}$ or 9 ; in a direction such that we have a P.D. of $90^{\circ}$ the intensity will be 1 , while with a P.D. of $120^{\circ}$ we have a triangle, there is no "closing side" and the intensity is zero. From now on it increases, attaining the value 1 again with a $\mathrm{H}$.D. of $180^{\circ}$

Fig. 1.

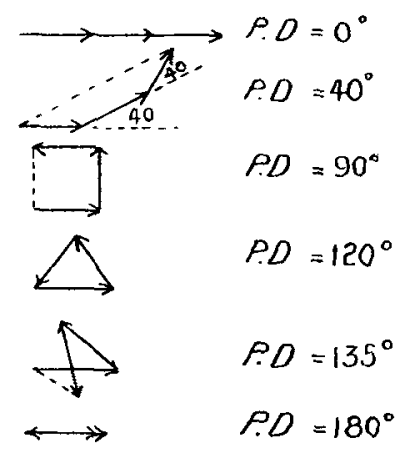

when the three vectors are superposed; two of these vectors cancel each other, the illumination being that due to the outstanding one. The various stages are shown in fig. 1 for 
of the Secondury Maxima of Grating Spectra.

different values of P.D. The first-order spectrum comes in such a direction that the P.D. is $360^{\circ}$ or the path difference is $\lambda$; consequently the point for which P.D. $=180^{\circ}$ is midway between the "central image" and the first spectrum, and the diffraction pattern is symmetrical about it. We thus see that there is a secondary maximum at this point, as shown in the lower part of fig. 1 .

From now on we shall only determine the positions of the minima when more than three lines operate. The complete curve can be calculated in the same manner.

In the case of a four-line grating we have intensity 16 at the centre, zero when the P.D. is $90^{\circ}$ or $180^{\circ}$, and unity when the P.D. is $120^{\circ}$. This gives us two secondary maxima between the principal maxima, their intensity being about $\frac{1}{14}$ that of the latter ; these maxima occur when the P.D. is $135^{\circ}$ and $225^{\circ}$. In fig. $2, I$ have given the positions of the

Fig. 2.

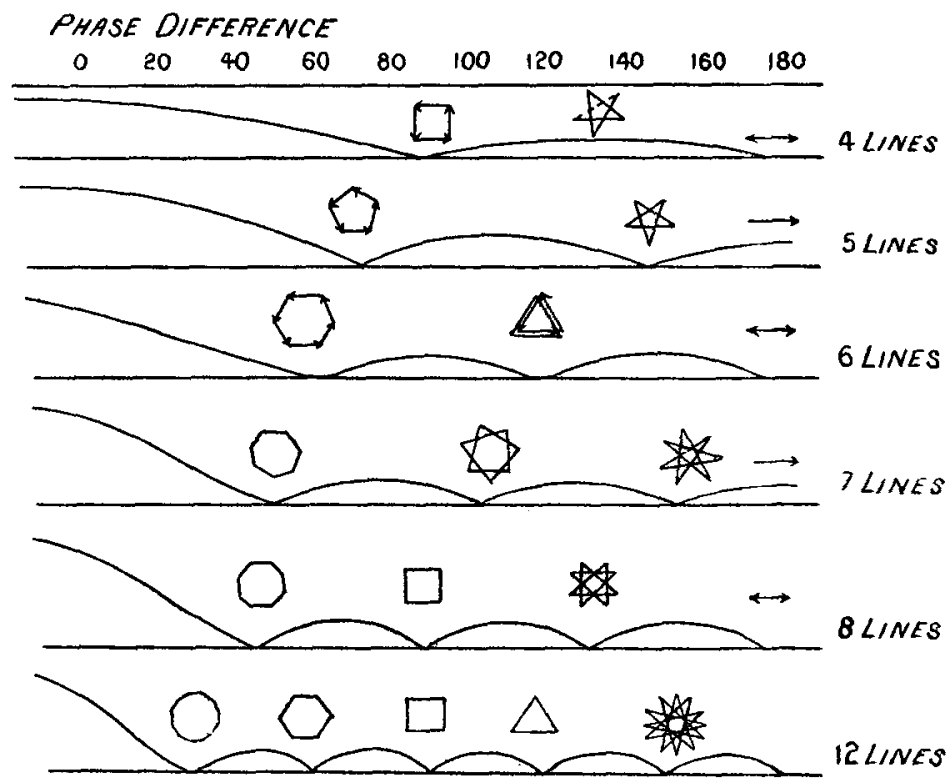

minima and the form of the closed amplitude figure at each, for gratings of 4, 5, 6, 7, 8, and 12 lines. The ordinates of the amplitude curves are not drawn to a scale of course.

With a five-line grating we get our first minimum when the five amplitude lines form a pentagon, the phase difference being $72^{\circ}$, and a second when they form a star, the phase 
difference in this case being $144^{\circ}$. At the centre $\left(180^{\circ}\right)$ we have intensity one, as in the case of the three-line grating.

It will be noticed that we have a zero value only when the starting and terminal points of our broken line of vectors coincide. With a phase difference of $180^{\circ}$ this will happen when the number of lines in the grating is even. It may also happen when a closed figure such as a triangle, square, or regular polygon is formed.

For a six-line grating we must show the existence of 4 secondary maxima. The illumination will be zero for the $180^{\circ}$ phase difference, also for that of $60^{\circ}$ when we bave a hexagon, and for $120^{\circ}$ when we have two superposed triangles. A star figure cannot be formed of 6 lines compounded as described.

In the case of a seven-line grating we have the zero minima for the regular polygon (P.D. 51 $\left.1^{\circ} 6\right)$ and for two star-shaped figures (P.D. $102^{\circ}$ and $154^{\circ}$ ), giving us five secondary maxima between the two principal maxima. The eight-line grating gives zero when the amplitude lines form an octagon, two superposed squares, an eight-pointed $\operatorname{star}\left(\right.$ P.D. $\left.=135^{\circ}\right)$ and also at the centre of symmetry (P.D. $=180^{\circ}$ ).

In the case of the twelve-line grating the lines form in snccession a twelve-sided polygon, two superposed hexagons, three squares, four triangles, and a twelve-pointed star, the phase difference in the latter case being $150^{\circ}$.

The following method of ascertaining the number of possible figures will be found useful.

Arrange around a circle as many equidistant dots as there are lines in the grating, and join the dots by straight lines, first shifting one dot, then two, three, four, \&c. With twelve dots we get the twelve-pointed star when we skip four dots each time. For a grating of thirteen lines we find it possible to form thirteen-pointed stars in five different ways, between $0^{\circ}$ and $180^{\circ}$ phase difference. Each of these gives zero jllumination, also the polygon of thirteen sides; consequently we have six minima between $0^{\circ}$ and $180^{\circ}$, or eleven secondary maxima. In the case of twelve dots we get but one star, skipping 1, 2, and 3 dots giving the hexagons, squares, and triangles.

We thus see that, in the case of a grating of $n$ lines, we have $(n-2)$ secondary maxima between the principal maxima, the intensity of which can be easily calculated from diagrams. similar to those given.

We will now consider the case of the optical grating with many thousand lines, and see what part the secondary maxima, which accompany the spectrun-lines, play. 
Our broken line now becomes essentially a smooth curve. We have our first minimum when it forms a complete circle, the phase difference between disturbances from the first and last lines being $360^{\circ}$, or the path difference $\lambda$. The first secondary maximum occurs when the line has wound up into a circle and a half. The ratio of the intensity of the secondary to that of the principal maximum is obviously the ratio of the square of the diameter of the circle of $1 \frac{1}{2}$ turns to the square of the total length of the line. This we easily find by winding up a strip of paper of known length. It will be found to be about $1: 23$.

This shows us that, no matter how many lines we have in the grating, our spectrum-lines will always be accompanied by close companions, baving at least $\frac{1}{23}$ of their brightness. For an eight-line grating the ratio is not very different, being about $\frac{1}{2} \hat{\imath}$. It occurs for a phase difference approximately such that we have a regular pentagon, three sides of which are made of double lines. For a four-line grating it is about ${ }_{16}^{\frac{1}{6}}$ (triangle with one side double) *, and for a three-line grating $\frac{1}{9}$. This last is the maximum value of the ratio.

It seems quite surprising that even for optical gratings the secondary maxima have a brilliancy very nearly one half of that which obtains in the case of a three-line grating. Their angular distance from the spectrum-lines is such as to make the path difference between disturbances coming from the first and last lines of the grating $\frac{3 \lambda}{2}$ more than the path difference at the line. This angle is obviously that subtended by one and one half waves, at a distance equal to the width of the ruled surface. The distance between the secondary maxima is thus seen to depend upon the width of the grating, and not upon the number of lines. At first sight there may appear to be some difficulty about this, sinee there are $n-2$ secondary maxima; and we might very naturally expect an increase of $n$ to push them nearer together. This is, however, only true when the "grating space" remains constant, $i$. e. when we add new lines of the same spacing.

Suppose we have a grating of given width with 20 'lines which gives 18 secondary maxima. If we interpolate lines between the lines already present, we double the number of secondary maxima to be sure; but the principal maxima (spectra.) of odd order disappear by interference; in other

* This, however, is not the centre or brightest point of the maximum, which is for a P.D. of $135^{\circ}$, the figure having the form shown in fig. 2 . It this point the intensity is about ${ }_{i=}$ that of the principal maxima. 
words, they are now twice as far apart as before, the spacing of the secondary maxima remaining the same.

The analogy between the secondary maxima and the fringes produced by a rectangular aperture of the same size as the ruled surface, can be studied to advantage by means of coarse gratings made by ruling four or five lines on a piece of smoked plate glass, and making the lower third of the grating clear by wiping out the lines. Sun or are light filtered through red glass should be used with a small spectrometer, the grating and aperture being covered in succession or used simultaneously.

XLVI. On the Figure of the Earth. By J. PrEscotT, M.A., Lecturer in Mathematics at the Manchester Sihool of Tecknology*.

THALL assume that the figure of the earth is an oblate spheroid of small ellipticity and I propose to find this ellipticity. I shall also assume that the figure is the same as it would be if the earth were wholly liquid. There can be little doubt that the figure obtained on these assumptions is not far from the actual shape. For, the earth's crust is probably not rigid enough to resist the forces pulling it into this shape.

It is necessary first to find the potential, at an external point on the axis, of a thin shell bounded by similar oblate spheroids whose generating curves are

$$
\begin{aligned}
& \frac{x_{1}^{2}}{a^{2}}+\frac{y_{1}{ }^{2}}{b^{2}}=1, \\
& \frac{x_{2}^{2}}{a^{2}}+\frac{y_{2}{ }^{2}}{b^{2}}=1+\sigma,
\end{aligned}
$$

where

$$
b^{2}(1+\sigma)=(b+\delta b)^{2}=b^{2}+2 b \delta b,
$$

or

$$
s=2 \frac{\delta b}{b}
$$

and $b$ is the polar semi-axis.

Let $\mathbf{P}$ be the external point, $O$ the centre of the shells, $\mathrm{OP}=r,(a-b)=e b, \mathrm{~K}=$ the gravitation constant, $\rho=$ density. The mass of a thin ring of the shell between the planes $y$ and $y+\delta y$ is

$$
\begin{aligned}
& \pi \rho\left(x_{2}^{2}-a_{1}^{2}\right) \delta y \\
= & \pi \rho \sigma a^{2} \delta y .
\end{aligned}
$$

* Communicated by the Author. 